\title{
Significant impact of amount of PCR input templates on various PCR-based DNA methylation analysis and countermeasure
}

\author{
Zhaojun Liu' ${ }^{1, *}$, Jing Zhou ${ }^{1,}{ }^{,}$, Liankun Gu ${ }^{1}$, Dajun Deng ${ }^{1}$ \\ ${ }^{1}$ Key Laboratory of Carcinogenesis and Translational Research (Ministry of Education/Beijing), Division of Etiology, Peking \\ University Cancer Hospital and Institute, Haidian District, Beijing, 100142, China \\ *These authors contributed equally to this work
}

Correspondence to: Dajun Deng, email: dengdajun@bjmu.edu.cn

Keywords: DNA methylation, false negative detection, MethyLight, DHPLC, MSP

Received: June 06, $2016 \quad$ Accepted: July 20, $2016 \quad$ Published: July 28, 2016

\section{ABSTRACT}

Methylation changes of CpG islands can be determined using PCR-based assays. However, the exact impact of the amount of input templates (TAIT) on DNA methylation analysis has not been previously recognized. Using COL2A1 gene as an input reference, TAIT difference between human tissues with methylation-positive and -negative detection was calculated for two representative genes GFRA1 and P16. Results revealed that TAIT in GFRA1 methylation-positive frozen samples $(n=332)$ was significantly higher than the methylation-negative ones $(n=44)(P<0.001)$. Similar difference was found in P16 methylation analysis. The TAIT-related effect was also observed in methylation-specific PCR (MSP) and denatured high performance liquid chromatography (DHPLC) analysis. Further study showed that the minimum TAIT for a successful MethyLight PCR reaction should be $\geq 9.4 \mathrm{ng}\left(\mathrm{Ct}_{\mathrm{COL2A1}} \leq \mathbf{2 9 . 3}\right)$, when the cutoff value of the methylated-GFRA1 proportion for methylation-positive detection was set at $1.6 \%$. After TAIT of the methylation non-informative frozen samples $\left(n=94 ; \mathrm{Ct}_{\text {COL2A1 }}>29.3\right)$ was increased above the minimum TAIT, the methylation-positive rate increased from $\mathbf{7 2 . 3} \%$ to $\mathbf{9 5 . 7 \%}$ for GFRA1 and $26.6 \%$ to $54.3 \%$ for $P 16$, respectively ( $P$ s $<0.001$ ). Similar results were observed in the FFPE samples. In conclusion, TAIT critically affects results of various PCR-based DNA methylation analyses. Characterization of the minimum TAIT for target CpG islands is essential to avoid false-negative results.

\section{INTRODUCTION}

Alterations of DNA methylation play undisputed roles in cancer development through epigenetic inactivation and reactivation of tumor-related genes [1-8]. Methylation information on $\mathrm{CpG}$ islands are persistently maintained in various kinds of stored samples, i.e., genomic DNA extracted from frozen tissues, formalin fixed paraffin embedded (FFPE) tissues, cell line pellets, and various body fluids, which provide rich sample resources for DNA methylation studies and biomarker development. The methylation status of individual cytosines in genomic DNA can be quantitatively determined using bisulfite-treated DNA templates combined with different PCR-based assays including methylation-specific PCR (MSP), MethyLight, Pyrosequencing, denaturing high performance liquid chromatography (DHPLC), and matrix-assisted laser desorption/ ionization time-of-flight mass spectrometry
(MALDI-TOF MS) [9-15]. Most importantly, alterations of DNA methylation, even if they occur only in a very limited number of cells in specimens, can be sensitively detected using methylation or unmethylation specific assays. These DNA methylation changes can be used as one kind of optimal biomarker for predicting disease development and progression [16, 17].

It is well known that the efficiency of bisulfitebased DNA methylation PCR is generally much lower than regular PCR using double-strand genomic DNA as templates due to a number of reasons. This includes DNA degradation during bisulfite treatment, low stability of single-strand DNA, and strand-specific PCR amplification. Hence, more templates should be used in the bisulfite-PCR assays. Different amplification protocols recommend the use of 50-500 ng of bisulfite-treated DNA [14]. These strategies are not feasible for most researches when very limited amounts of DNA are available for PCR amplification, such as DNA samples extracted from 
endoscope biopsy, serum or plasma, and other body fluids. The variability in DNA methylation analysis may result from stochastic PCR amplification that often occurs at low template concentration and leads to false-negative detection [10]. Unfortunately, not enough attention has been paid to the false-negative events that may frequently occur when the amount of input template (TAIT) is lower than the detection limit, the minimum TAIT for a successful PCR reaction. Moreover, the exact impact of TAIT on outcomes of DNA methylation analysis has not been systematically studied. In the present study, we analyzed the influence of TAIT on the bisulfite-PCR-based DNA methylation analysis and provided a countermeasure to avoid the false-negative phenomenon.

\section{RESULTS}

\section{Significant impact of TAIT on outcomes of DNA methylation analysis by MethyLight}

We used the average $\mathrm{Ct}$ value for the reference gene COL2A1 as an indicator of TAIT level for a sample in MethyLight analysis. Through re-analyzing our previously published MethyLight data for FFPE surgical samples from Korean patients with gastric carcinoma (Set-1, Table 1) [5], we found that TAIT level in GFRA1 methylationpositive samples $(n=62)$ was significantly higher than the methylation-negative ones $(n=58)\left(\mathrm{Ct}_{\mathrm{COL} 2 \mathrm{~A} 1}[\right.$ median $]$ : 31.0 vs. 32.9, $\Delta \mathrm{Ct}=1.9 ; P<0.001$; Figure $1 \mathrm{~A})$. To validate the TAIT difference, we analyzed another set of surgical gastric carcinoma FFPE samples (Set-2) using MethyLight, and observed the same phenomenon: the average TAIT level in GFRA1 methylation-positive and -negative FFPE samples ( $n=11$ and 86 ) were 31.5 and 33.0, respectively $(\Delta \mathrm{Ct}=1.5 ; P=0.002$, Figure 1B). Similarly, in oral mucosa biopsy FFPE samples (Set-3), TAIT level in P16 methylation-positive oral biopsy FFPE samples $(n=44)$ was also much higher than the methylation-negative ones $(n=107)(29.6 v s .31 .4, \Delta \mathrm{Ct}=1.8 ; P<0.001$, Figure 1C). Unexpectedly, such differences were also found in DNA methylation analysis using frozen gastric surgical samples (Set-4): TAIT level in GFRA1 methylation-positive samples $(n=332)$ was significantly higher than the methylation-negative ones $(n=44)$ (29.6 vs. 32.0, $\Delta \mathrm{Ct}=$ $2.4 ; P<0.001$, Figure 1D). Similarly, TAIT level in $P 16$ methylation-negative samples $(n=196)$ was lower than the methylation-positive ones $(n=180)$ either (30.5 vs. 29.1, $\Delta \mathrm{Ct}=1.4 ; P<0.001$, Figure 1E). Together, these results indicate that TAIT level in the MethyLight assay may significantly affect results of DNA methylation detection. It is possible that false-negative results occur when the template molecules available for PCR amplification in tested samples are very low, or when TAIT level is lower than the minimum TAIT for a successful PCR reaction.

It is well recognized that the quality of genomic DNA extracted from FFPE samples is lower than frozen samples because of DNA degradation in formalin fixating, paraffin embedding, and deparaffinage processes. This phenomenon is consistently displayed in the MethyLight analysis. The $\mathrm{Ct}_{\mathrm{COL} 2 \mathrm{~A} 1}$ value for most FFPE samples (Set-1, -2 , and -3 ) was more than 29.3 , the minimum TAIT value described below; in contrast, for most frozen samples (Set-4 and -6 ), $\leq 29.3$ (Table 1, the last column). This indicates that the false-negative detection may frequently occur in MethyLight analysis using FFPE samples without adjustment of input DNA.

\section{Characterization of the minimum TAIT for successful MethyLight analyses}

To characterize inference of unmethylated counterparts and the minimum amount (or detection limit) of methylated DNA templates for a success MethyLight reaction, a GFRA1-methylated template solution array was prepared through serial dilutions with GFRA1unmethylated DNA. The bisulfite-treated DNA working solution $(0.7 \mathrm{ng} / \mu \mathrm{L})$ from RKO cells was initially diluted with another bisulfite-treated DNA stock solution (at equal concentration) from GES1 cells, and further diluted with TE buffer to various proportions (Table 2). $1.7 \mu \mathrm{L}$ of these diluted template solutions was added into each MethyLight reaction (final volume, $15 \mu \mathrm{L}$; triplicate). Results showed that the $\mathrm{Ct}$ value for the methylatedGFRA1 CpG islands $\left(\mathrm{Ct}_{\mathrm{GFRA}}\right)$ gradually increased along with decrease in the amounts of DNA from RKO cells, whether diluted with DNA from GES1 cells or TE buffer. When the amount of the DNA from RKO cells was $\geq 0.15$ ng per PCR reaction, the $\mathrm{Ct}_{\text {GFRA1 }}$ value was consistently determined in the entire triplicate in the template array. Thus, $0.15 \mathrm{ng}$ bisulfite-treated DNA is defined as the minimum amounts of template DNA from GFRA1 methylated cells. Interestingly, along with increase in the amounts of GFRA1-unmethylated DNA from GES1 cells, the exact $\mathrm{Ct}_{\text {GFRA1 }}$ values for the same amount of the DNA from RKO cells were not increased, indicating no interference resulted from the occurrence of unmethylated GFRA1 templates. We also found that methylation signal for the methylated P16 was also consistently detected when $0.15 \mathrm{ng}$ bisulfite-treated DNA was added into each PCR reaction, suggesting a similar efficiency of PCR amplification for methylated GFRA1 and P16 templates.

\section{Strategy to avoid false negative results in DNA methylation analysis}

Generally, both methylated and unmethylated alleles exist in human tissues due to the heterogeneous cell composition. Despite imprinting of genes, the proportion of methylated alleles for $\mathrm{CpG}$ islands in disease-related genes in tissues may greatly change as adaptations to various environmental factors occur. This is particularly true, especially in biopsies from pathological lesions 
comparing with normal tissues. Therefore, characterization of the minimum amount of total input genomic DNA for a successful methylation analysis is a critical issue to avoid false negative results. According to results from the above minimum TAIT for GFRA1 and P16 methylation analyses, when the cutoff value for the proportion of methylated GFRA1 or P16 alleles in a tested sample is set at $1.6 \%$, in order to avoid false negative results, at least $9.4 \mathrm{ng}$ bisulfite-treated input DNA $\left(\mathrm{Ct}_{\mathrm{COL} 2 \mathrm{Al}} \leq 29.3\right)$ should be added into each PCR reaction. To evaluate the false negative rate in the methylation non-informative samples $\left(\mathrm{Ct}_{\mathrm{COL} 2 \mathrm{Al}}>29.3\right)$ in previous methylation analysis, we re-analyzed the methylation status of the representative genes using increased TAIT $\left(\mathrm{Ct}_{\mathrm{COL} 2 \mathrm{~A} 1} \leq 29.3\right)$ as described in details below.

Among Set-2 and Set-4, 96.9\% (94/97) FFPE samples and $25.0 \%$ (94/376) frozen samples were detected with $\mathrm{Ct}_{\text {COL2A1 }}>29.3$ (Table 1, the last column), suggesting the GFRA1 methylation status may be underestimated due to lower TAIT level. Therefore, the methylation status of GFRA1 in these samples was re-analyzed using more input template $\left(\mathrm{Ct}_{\mathrm{COL} 2 \mathrm{~A} 1} \leq 29.3\right)$ and MethyLight assay. Results revealed that the GFRA1 methylation-positive rate was significantly increased from $10.6 \%$ to $39.4 \%(P<0.001)$ for these 94 FFPE samples. For the 94 frozen samples, the GFRA1 methylation-positive rate also significantly increased from $72.3 \%$ to $95.7 \%(P<0.001)$. Similarly, $P 16$ methylation-positive rate increased from $26.6 \%$ to $54.3 \%$ $(P<0.001)$. Subsequently, no significant difference of TAIT levels was found between the methylation-positive and -negative samples in the re-analysis. The average TAIT level in the GFRA1 methylation-positive FFPE samples $(n=37)$ was not significantly higher than the methylationnegative ones $(n=57)\left(\mathrm{Ct}_{\mathrm{COL} 2 \mathrm{~A} 1}[\right.$ median]: 28.5 vs. 29.0,

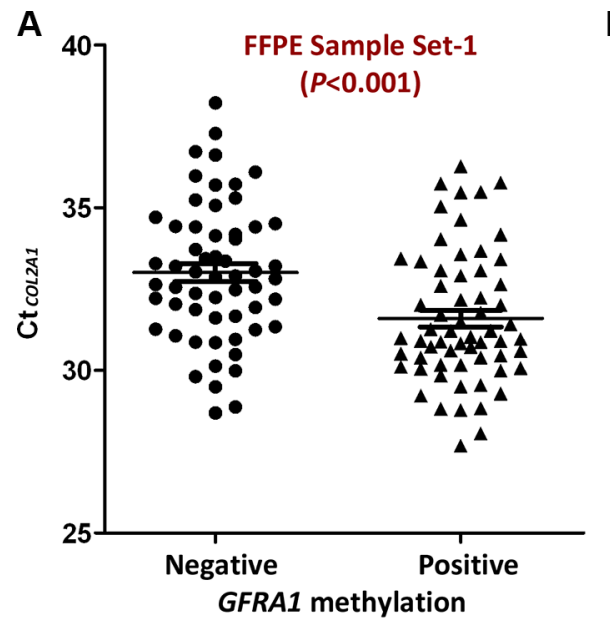

D

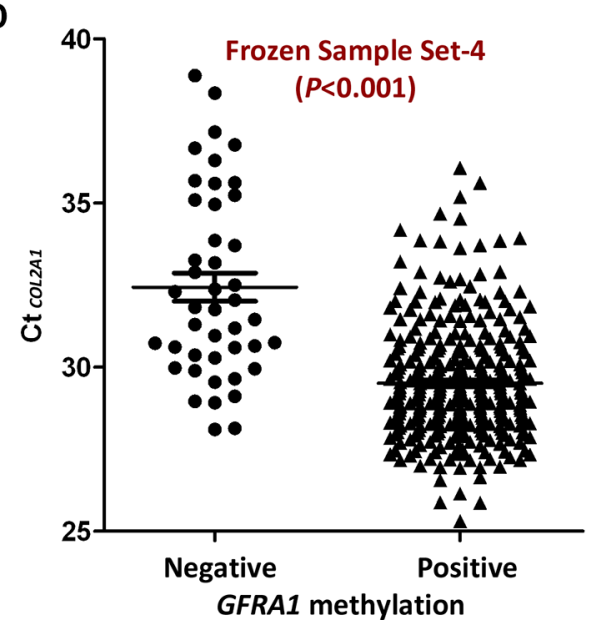

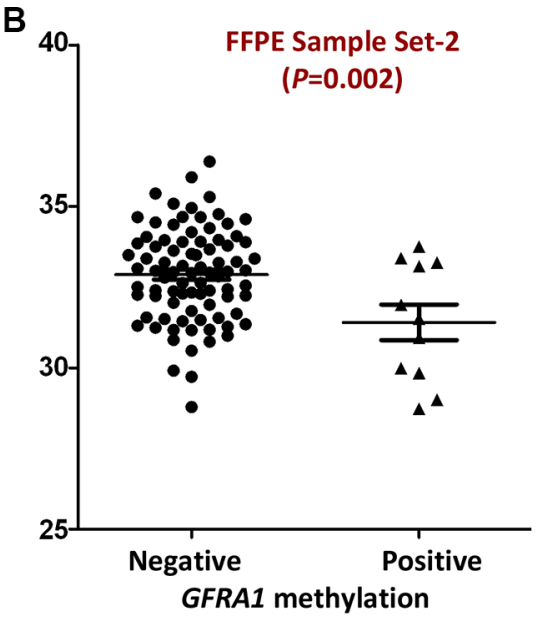
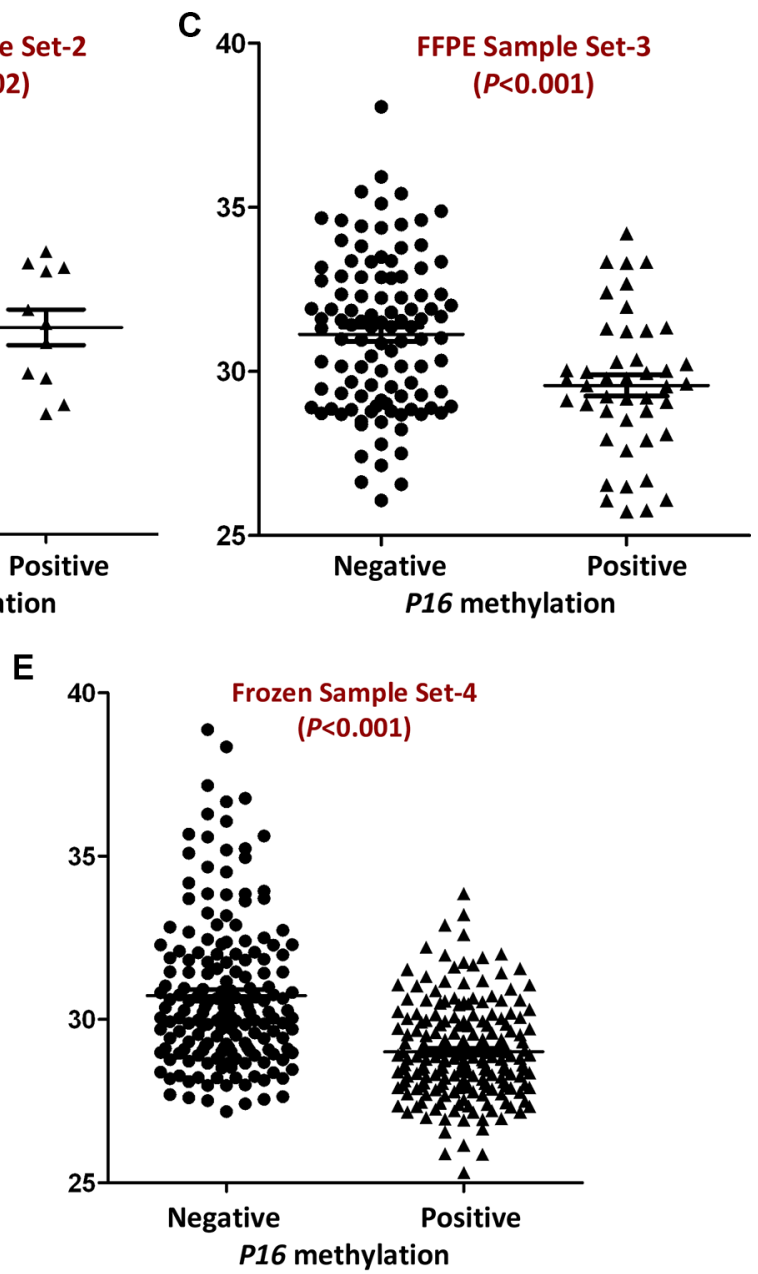

Figure 1: Comparison of $\mathrm{Ct}_{\mathrm{COL2A1}}$ in GFRA1 or P16 methylation positive samples and methylation negative samples. (A and B) The amount of input templates (TAIT) in surgical gastric carcinoma FFPE samples from Korean and Chinese, respectively. GFRA1 methylation positive and negative: with or without methylation signal in MethyLight analysis; (C) TAIT in the oral mucosa biopsy FFPE samples with or without P16 methylation signal in MethyLight analysis; (D and E) TAIT in surgical gastric carcinoma frozen samples with or without GFRA1 or P16 methylation signal in MethyLight analysis, respectively. The mean and SE values were marked with lines. 


\begin{tabular}{|c|c|c|c|c|c|c|c|}
\hline Set & Subjects & Storage & Organ & Pathological changes & $n$ & $\begin{array}{l}\text { Methylation in } \\
\text { gene, assay }\end{array}$ & $\begin{array}{c}\text { Proportion of } \mathrm{Ct}_{\mathrm{COL2A1}} \leq 29.3 \\
\text { samples }^{1}\end{array}$ \\
\hline Set-1 & Korean & FFPE & Stomach & Adenocarcinoma & 120 & $\begin{array}{c}\text { GFRA1, } \\
\text { MethyLight }\end{array}$ & $13.3 \%$ \\
\hline Set-2 & Chinese & FFPE & Stomach & Adenocarcinoma & 97 & $\begin{array}{c}\text { GFRA1, } \\
\text { MethyLight }\end{array}$ & $3.1 \%$ \\
\hline Set-3 & Chinese & FFPE & Oral & Epithelial dysplasia & 151 & P16, MethyLight & $33.8 \%$ \\
\hline Set-4 & Chinese & Frozen & Stomach & $\begin{array}{c}\text { Adenocarcinoma } \\
\text { or corresponding normal }\end{array}$ & 376 & $\begin{array}{l}\text { GFRA1 \& P16, } \\
\text { MethyLight }\end{array}$ & $75.5 \%$ \\
\hline Set-5 & Chinese & FFPE & Stomach & Epithelial dysplasia & 116 & $P 16, \mathrm{MSP}$ & $70.7 \%$ \\
\hline Set-6 & Chinese & Frozen & Colon & $\begin{array}{c}\text { Adenocarcinoma } \\
\text { or corresponding normal }\end{array}$ & 185 & GFRA1, DHPLC & $90.8 \%$ \\
\hline
\end{tabular}

${ }^{1}$ The value for the reference gene in MethyLight analysis without adjustment of input DNA.

$\Delta \mathrm{Ct}=0.5 ; P=0.070 ;$ Figure $2 \mathrm{~A}$ ), and the average TAIT level in the GFRA1 or P16 methylation-positive frozen samples ( $n=90$ or 51$)$ was similar with their methylationnegative samples $(n=4$ or 43$)\left(\mathrm{Ct}_{\mathrm{COL} 2 \mathrm{~A} 1}\right.$ [median]: $28.7 \mathrm{vs}$. 28.9, $\Delta \mathrm{Ct}=0.2, P=0.481$ for $G F R A 1$, Figure 2B; $28.6 v s$. $28.9, \Delta \mathrm{Ct}=0.3, P=0.053$ for $P 16$, Figure $2 \mathrm{C}$ ).

\section{Evaluation of false negative prevalence in other PCR-based methylation assays}

We wonder whether TAIT level also affects results of other PCR-based methylation assays. The amounts of template DNA in Set-5 and Set-6 samples (Table 1) were detected using MethyLight at first. The P16 and GFRA1 methylation were analyzed using MSP and DHPLC, respectively. Because the reported detection limit $(0.1-0.4 \%)$ for MSP and DHPLC assays [13, 18] was similar to MethyLight (Table 1), we directly used the minimum TAIT value for MethyLight to classify the methylation informative and not informative samples in MSP and DHPLC analysis. For 34 of 116 FFPE samples $(29.3 \%)$ in Set-5, TAIT values in MSP analysis were lesser than the minimum TAIT $\left(\mathrm{Ct}_{\mathrm{COL} 2 \mathrm{Al}}>29.3\right)$. The P16 methylation-positive rate in 82 informative samples $\left(\mathrm{Ct}_{\mathrm{COL} 2 \mathrm{~A} 1} \leq 29.3\right)$ was significantly higher than that in the 34 not informative samples $\left(\mathrm{Ct}_{\mathrm{COL} 2 \mathrm{~A} 1}>29.3\right)(78.0 \%$ vs. $47.1 \%, P<0.001)$. Similarly, for 17 of 185 frozen samples $(9.2 \%)$ in Set-6, TAIT values in the PCR amplification step in DHPLC analysis were less than the minimum TAIT $\left(\mathrm{Ct}_{\mathrm{COL} 2 \mathrm{~A} 1}>29.3\right)$. The GFRA1 methylation-positive rate in 168 informative samples was also higher than that in the not informative samples $(58.3 \%$ vs. $41.2 \%)$, but not significant $(P=0.174)$. These results indicate false negative results do occur when the amount of input DNA in MSP or DHPLC analysis is less than the minimum value.

\section{DISCUSSION}

Various PCR assays for DNA methylation analysis have been developed using bisulfite-treated genomic DNA as PCR template [9-15]. Poor quality and low quantity of input DNA are always important parameters to decrease PCR efficiency that may lead to methylation non-informative (false negative) result. This is becoming a serious problem as more researchers become interested in DNA methylation in both basic and translational research. In the present study, we have systematically evaluated the exact influence of TAIT on the detection of the methylation status of $\mathrm{CpG}$ islands in two representative genes GFRA1 and P16 using MethyLight assay, and found that TAIT is a critical factor that leads to a relative proportion of false negative results, especially in FFPE samples. Meanwhile, we have also provided a strategy to avoid the false negative results through determining the minimum TAIT value (detection limitation) and using more input DNA than the minimum TAIT.

It has previously been reported that the number of molecules available for PCR amplification is critical for accurate characterization of methylation ratios using MALDI-TOF MS assay [15]. When few molecules are present, the reaction can still be performed successfully, but the sampling error has a devastating effect on the confidence level of the quantitative results in a representative sample. Unfortunately, enough attention has still not been paid to the TAIT-related false negative in most of studies. In one of our recent works, we used the minimum TAIT strategy to avoid false negative results in P16 methylation analysis in a multicenter prospective study [2]. Here, we have described for the first time the impact of TAIT on outcomes of DNA methylation analysis and describe how to overcome this obstacle in details. 
Table 2: Ct values for the input reference $C O L 2 A 1$ and $G F R A 1$ in various bisulfite-treated DNA samples from RKO cells serially diluted with TE buffer and/or bisulfite-treated DNA from GES1 cells in MethyLight analysis

\begin{tabular}{|c|c|c|c|c|c|c|c|}
\hline \multicolumn{2}{|c|}{ RKO-DNA diluted by } & \multicolumn{2}{|c|}{$\begin{array}{l}\text { Total amounts of input } \\
\text { DNA from RKO \& GES1 }\end{array}$} & \multicolumn{4}{|c|}{ Net amounts of DNA templates from RKO cells } \\
\hline GES1-DNA & TE-Buffer & ng/reaction & $\mathrm{Ct}_{\text {COL2A1 }}$ & ng/reaction & Percentage $^{a}$ & $\mathrm{Ct}_{\text {GFRA1 }}$ & $\mathbf{C t}_{\mathrm{P} 16}$ \\
\hline- & - & 18.78 & 27.90 & 1.18 & $6.3 \%$ & 32.82 & 33.58 \\
\hline $1 / 2$ & - & 18.78 & 28.18 & 0.59 & $3.1 \%$ & 33.92 & 35.04 \\
\hline $1 / 4$ & - & 18.78 & 27.93 & 0.29 & $1.6 \%$ & 34.78 & 35.13 \\
\hline $1 / 8$ & - & $\underline{18.78}$ & $\underline{28.18}$ & $\underline{0.15}$ & $\underline{0.8 \%}$ & $\underline{35.84}$ & $\underline{36.61}$ \\
\hline $1 / 16$ & - & 18.78 & 28.09 & 0.07 & $0.4 \%$ & $37.38^{1}$ & 37.76 \\
\hline $1 / 32$ & - & 18.78 & 28.05 & 0.04 & $0.2 \%$ & $38.25^{1}$ & $38.49^{1}$ \\
\hline $1 / 64$ & - & 18.78 & 28.10 & 0.02 & $0.1 \%$ & $38.99^{1}$ & $38.87^{1}$ \\
\hline- & $1 / 2$ & 9.39 & 29.34 & 0.59 & $6.3 \%$ & 34.16 & 34.70 \\
\hline $1 / 2$ & $1 / 2$ & 9.39 & 29.35 & 0.29 & $3.1 \%$ & 35.35 & 36.21 \\
\hline $1 / 4$ & $1 / 2$ & $\underline{9.39}$ & $\underline{29.33}$ & $\underline{0.15}$ & $\underline{1.6 \%}$ & $\underline{36.15}$ & $\underline{\mathbf{3 7 . 3 0}}$ \\
\hline $1 / 8$ & $1 / 2$ & 9.39 & 29.41 & 0.07 & $0.8 \%$ & $37.41^{1}$ & 38.07 \\
\hline $1 / 16$ & $1 / 2$ & 9.39 & 29.53 & 0.04 & $0.4 \%$ & 39.84 & 38.63 \\
\hline $1 / 32$ & $1 / 2$ & 9.39 & 29.38 & 0.02 & $0.2 \%$ & $37.95^{1}$ & $38.74^{1}$ \\
\hline $1 / 64$ & $1 / 2$ & 9.39 & 29.26 & 0.01 & $0.1 \%$ & $36.83^{1}$ & Undet $^{2}$ \\
\hline- & $1 / 4$ & 4.70 & 30.73 & 0.29 & $6.3 \%$ & 35.28 & 36.78 \\
\hline $1 / 2$ & $1 / 4$ & $\underline{4.70}$ & $\underline{31.08}$ & $\underline{0.15}$ & $\underline{3.1 \%}$ & $\underline{37.59}$ & $\underline{37.73}$ \\
\hline $1 / 4$ & $1 / 4$ & 4.70 & 31.10 & 0.07 & $1.6 \%$ & 39.17 & $38.18^{1}$ \\
\hline $1 / 8$ & $1 / 4$ & 4.70 & 30.64 & 0.04 & $0.8 \%$ & $38.12^{1}$ & $37.71^{1}$ \\
\hline $1 / 16$ & $1 / 4$ & 4.70 & 30.95 & 0.02 & $0.4 \%$ & $39.74^{1}$ & Undet $^{2}$ \\
\hline $1 / 32$ & $1 / 4$ & 4.70 & 30.78 & 0.01 & $0.2 \%$ & Undet $^{2}$ & Undet $^{2}$ \\
\hline $1 / 64$ & $1 / 4$ & 4.70 & 30.83 & 0.005 & $0.1 \%$ & 37.96 & Undet $^{2}$ \\
\hline- & $1 / 8$ & $\underline{2.35}$ & $\underline{31.61}$ & $\underline{0.15}$ & $\underline{6.3 \%}$ & $\underline{36.67}$ & $\underline{\mathbf{3 7 . 1 0}}$ \\
\hline $1 / 2$ & $1 / 8$ & 2.35 & 32.04 & 0.07 & $3.1 \%$ & $37.45^{1}$ & $38.10^{1}$ \\
\hline $1 / 4$ & $1 / 8$ & 2.35 & 31.93 & 0.04 & $1.6 \%$ & 38.14 & $38.48^{1}$ \\
\hline $1 / 8$ & $1 / 8$ & 2.35 & 31.81 & 0.02 & $0.8 \%$ & $37.96^{1}$ & Undet $^{2}$ \\
\hline $1 / 16$ & $1 / 8$ & 2.35 & 31.92 & 0.01 & $0.4 \%$ & $41.02^{1}$ & $38.93^{1}$ \\
\hline $1 / 32$ & $1 / 8$ & 2.35 & 31.64 & 0.005 & $0.2 \%$ & Undet $^{2}$ & Undet $^{2}$ \\
\hline $1 / 64$ & $1 / 8$ & 2.35 & 31.62 & 0.002 & $0.1 \%$ & Undet $^{2}$ & Undet $^{2}$ \\
\hline
\end{tabular}

The underlined values indicate the total amount of input DNA, $\mathrm{Ct}_{\text {COL2A1/GFRA1/P16 }}$ when the minimum net amount of RKO cell DNA ( $0.15 \mathrm{ng} /$ reaction) was added into each MethyLight reaction; The values in red characters are various $\mathrm{Ct}$ values when $0.15 \mathrm{ng}$ of RKO DNA was presented in $9.39 \mathrm{ng}$ of input DNA (1.6\%) in each MethyLight reaction. Ct value is written in bold characters when informative methylation signal is always determined in triplicate.

${ }^{1} \mathrm{Ct}_{\text {GFRA1 }}$ value is undetermined in one or two tubes in triplicate.

${ }^{2} \mathrm{Ct}_{\text {GFRA1 }}$ value is undetermined in all of triplicate.

We have not only observed the impact of TAIT on outcome of DNA methylation analysis using MethyLight assay, but also observed the same phenomenon using MSP and DHPLC assay. The TAIT-related inference might be applicable for other PCR-based methylation assays such as
Pyrosequencing and COBRA. The remarkable effect of the number of input templates available for PCR amplification in MALDI-TOF MS assay supports this extrapolation [15]. The amount of input DNA for only 17 of 185 Set-6 samples was lesser than the minimum TAIT in the PCR 
amplification step in DHPLC analysis. This may account for that the difference of GFRA1 methylation positive rate between the informative and not informative samples was not significant.

PCR amplification efficiency is amplicon sequencedependent. Although we found the minimum TAIT level for GFRA1 was not different from P16, we recommend determining the TAIT value for each interested amplicon in $\mathrm{CpG}$ islands, especially for these genes with different copy number in the genome. Because commercial standards for methylated and unmethylated DNA substances are not available at present, we have to use the genomic DNA from cancer cell lines as the homogenously methylated and unmethylated reference DNA to obtain standard curves in MethyLight analysis. Application of artificially and completely methylated genomic DNA sample by M.SssI DNA methyltransferase may be an alternative. Although the artificial DNA sample cannot be prepared as fresh or FFPE tissue controls, we recommend regularly using standard methylated and unmethylated DNA controls during DNA extraction, purification, bisulfite modification, and PCR processes to monitor the recovery, conversion, and amplification rates.

The minimum TAIT value may be different from the cutoff value in clinical practices. Clinical cancer tissues are mixtures of tumor cells and normal cells. A tumor may contain different cell sub-populations with various genetic and epigenetic changes. For a sample composed of high fraction of cancer cells with homogenous target DNA methylation changes (target cells), the minimum TAIT value may be lower than a sample composed of low fraction of target cells. Although the absolute amount of DNA template is the same for different kinds of samples, but the cutoff value for the proportion of methylated $\mathrm{CpG}$ islands may be sample- or gene-dependent. For example, in one of our prospective studies to predict malignant transformation risk of precancerous lesion epithelial dysplasia, the cutoff value for the proportion of P16-methylated cells was set at $1.6 \%$ to define $P 16$ methylation-positive samples and avoid false negative detections [2]. In another study on gastric cancer metastasis, GFRA1 methylation signal was detected in $60.2 \%$ cancer tissue samples from the discovery cohort, a cutoff value for the proportion of GFRA1-methylated cells $(>26.4 \%$ ) was further calculated according to ROC curve to define GFRA1 methylation-positive samples in the Kaplan-Meier survival analysis [5]. The minimum TAIT value can be used to avoid false negative detection; the cutoff value can be used to subclass samples with different prognosis or therapy sensitivity.

The minimum TAIT value was calculated based on both the absolute amount of genomic DNA containing fully methylated target alleles in input template and the total amounts of available templates in most frozen samples at good quality. The P16 methylation signal could be consistently determined when 0.15 ng (or more) P16methylated DNA was added into each PCR reaction. In order to enable most DNA samples methylation-informative after adjustment of input DNA, we selected $1.6 \%$ as the cutoff proportion of methylated P16 to definite a P16 methylation-positive sample (the minimum required total DNA, 38 ng; 9.4 ng/MethyLight reaction, two reactions for methylated-P16 and two reactions for the COL2A1 reference). When the clinical cutoff value is much higher than the detection limitation (i.e. GFRA1 methylation), the minimum TAIT value could be reduced further.
A

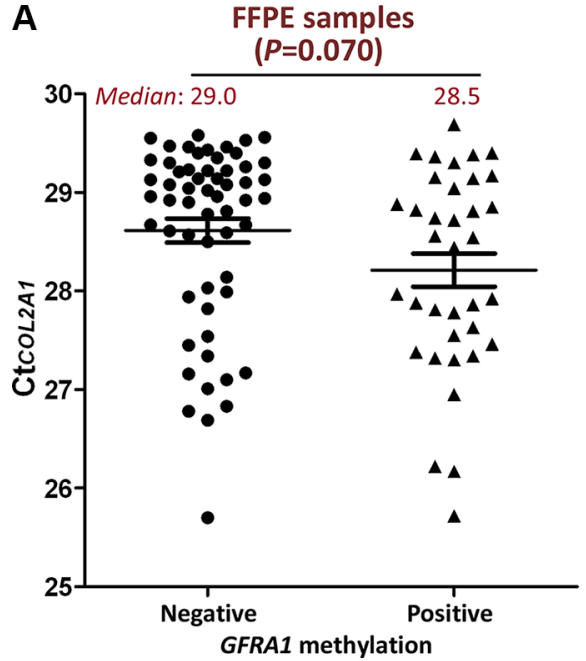

B

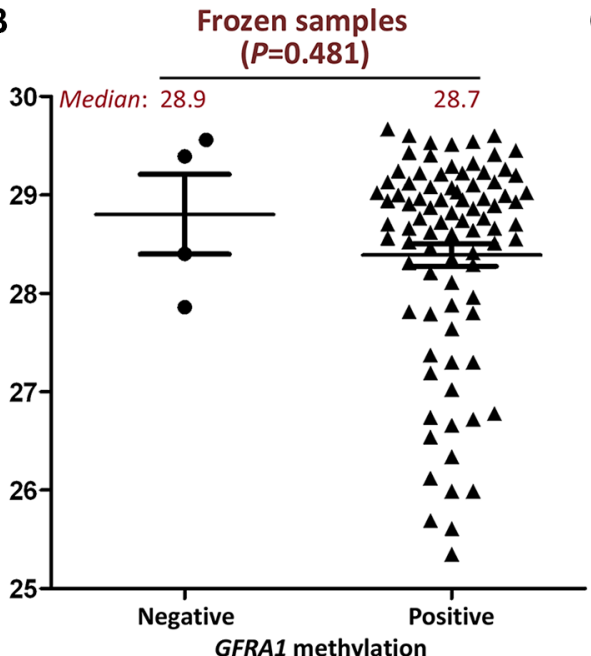

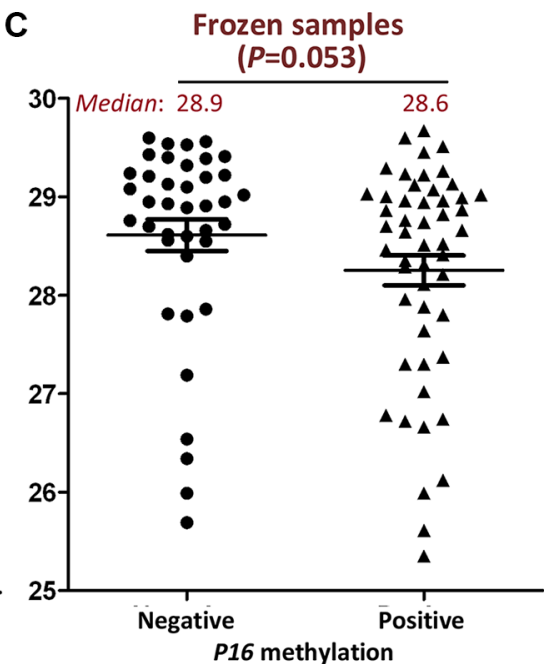

Figure 2: Comparison of $\mathrm{Ct}_{\mathrm{COL2A1}}$ in GFRA1 or P16 methylation positive re-analyzed samples and methylation negative ones. The amount of input templates (TAIT) in each re-analyzed sample was increased to above the minimum TAIT (Ct COL2AI $\leq 29.3)$ according to the $\mathrm{Ct}_{\mathrm{COL2A}}$ value obtained in the first-round analysis illustrated in Figure 1. (A and B) TAIT in surgical gastric carcinoma FFPE and frozen samples, respectively. GFRA1 methylation positive and negative: with or without methylation signal in MethyLight analysis; (C) TAIT in surgical gastric carcinoma frozen samples with or without P16 methylation signal in MethyLight analysis. The mean and SE values were marked with lines. The median values were also inserted. 
Except to increase the amount of input DNA, a quantitative nested approach (if available) may be an alternative way to avoid false negative detection. However, a proper negative control should be used to avoid falsepositive detection, which often occurs in qualitative nested analyses. For example, one can use a DNA sample containing $<1.6 \%$ methylated-P16 as a negative control if the cutoff proportion for the methylation-positive is set at $1.6 \%$.

In addition, because the $\mathrm{Ct}_{\mathrm{COL} 2 \mathrm{~A} 1}$ value not only represents the amount of input templates, but also represents the quality of input DNA and efficiency of bisulfite conversion of unmethylated cytosine residues, we recommend using the $\mathrm{Ct}_{\mathrm{COL2A}}$ value as an ideal TAIT reference to avoid false-negative events.

In conclusion, TAIT significantly impacts on outcomes of DNA methylation analysis using PCR-based assays. The minimum TAIT should be determined for each amplicon sequence in genomic DNA extracted from different kinds of samples to avoid false negative results.

\section{MATERIALS AND METHODS}

\section{Cell line and culture}

GFRA1 and P16 fully methylated cell line RKO and unmethylated cell line GES1 were kindly provided by Dr. Guoren Deng at University of California San Francisco and Dr. Yang Ke at Peking University Cancer Hospital/ Institute respectively. Cell lines were identified by STR analysis before use [5]. Cells were cultured in RPMI-1640 and $10 \%$ FBS.

\section{Human gastric mucosa samples (Table 1)}

Set-1 and Set-2 gastric carcinoma FFPE samples consist of 120 specimens from Seoul National University Hospital and 97 specimens from Peking University Beijing Cancer Hospital, respectively. For Korean sample Set-1, GFRA1 methylation data has been published in [5]. Set-3 samples consist of 151 oral epithelial dysplasia FFPE samples from Peking University School of Stomatology, Capital Medical University School of Stomatology, and Fourth Military Medical University Hospital of Stomatology collected between 2009 and 2011 in a fully anonymized and de-identified form, which were part of samples used in a multicentre prospective study (trial number NCT01695018, available at http://ClinicalTrials. gov). For those samples, P16 methylation data has been published in [2]. Set-4 frozen primary gastric carcinoma surgical tissues and their corresponding surgical margin samples $(n=376)$ were from 188 patients who underwent surgical treatment at Peking University Cancer Hospital \& Institute. These 188 patients were recruited in an ongoing prospective study (trial number NCT02159399, available at http://ClinicalTrials.gov). Set-5 samples consist of 116 gastric epithelial dysplasia FFPE samples from Peking University Beijing Cancer Hospital. Set-6 samples consist of 185 frozen colon adenocarcinoma samples from the same Hospital. These studies were approved by the local Institution Review Boards (IRB) at Peking University Beijing Cancer Hospital covered the collection and research use of tissues from all sites. All patients were given written informed consent unless the IRB permitted a waiver.

\section{DNA extraction and bisulfite modification}

Genomic DNA was extracted from cell line and frozen tissue samples using phenol/chloroform method. Briefly, paraffin-embedded tissues were treated with xylene and digested with proteinase K to obtain DNA [19]. The unmethylated-cytosine bases in the genomic DNA were converted to uracil bases by addition of $5 \mathrm{~mol} / \mathrm{L}$ of sodium bisulfite at $50^{\circ} \mathrm{C}$ overnight [20].

\section{Quantification of DNA}

A Nanodrop ND-1000 spectral photometer (Nanodrop Technologies, Wilmington, DE, USA) was used to detect DNA concentration. For calculation of the DNA concentration a multiplication factor of 33 was used for single-stranded DNA (bisulfite-DNA), and 50 for double-stranded DNA (genomic DNA).

\section{Preparation of methylated bisulfite-DNA solution serials}

Stock solution of bisulfite-DNA from RKO cells $(10 \mathrm{ng} / \mu \mathrm{L})$ was serially diluted at different ratios $(1: 0$, $1: 1,1: 3,1: 7,1: 15,1: 31$, and $1: 63)$ through adding stock solution of bisulfite-DNA from GES1 cells $(10 \mathrm{ng} / \mathrm{mL})$. These solutions were further diluted with TE buffer at ratios $1: 0,1: 1,1: 3$, and 1:7 to prepare various working solutions. All of these working solutions were used in MethyLight assay in triplicate through adding $1.7 \mu \mathrm{L}$ working solution into PCR mix (total volume $15 \mu \mathrm{L}$ ).

\section{MethyLight}

MethyLight assay was performed as previously described to detect the proportion of methylated $\mathrm{CpG}$ islands within P16 (115bp) and GFRA1 (158bp), respectively $[2,5]$. Gene-specific probes labeled with 6FAM and TAMRA were employed to quantify the relative copy number of methylated alleles compared to the COL2A1 reference (91 bp) [21]. When methylation signal for target $\mathrm{CpG}$ islands was consistently detected, it was defined as methylation-positive (without use of cutoff value). The average $\mathrm{Ct}$ value for the COL2A1 reference was used as an indicator of the TAIT level for a sample. 


\section{Methylation-specific PCR (MSP)}

The 150 bp methylated $P 16$ amplicon was analyzed used in the regular MSP analysis as described previously $[22,23]$.

\section{Denatured high performance liquid chromatography (DHPLC)}

The 522 bp methylated GFRA1 amplicon was analyzed used DHPLC and fluorescence detector as described previously [5].

\section{Statistical analysis}

Pearson's Chi-square test was used to compare categorical variables. The Mann-Whitney $U$-test and Student's $t$-test were used to compare the $\mathrm{Ct}$ value of COL2A1 $\left(\mathrm{Ct}_{\mathrm{COL2A1}}\right)$ between methylation-positive and -negative samples. All statistical tests were two-sided, and $P<0.05$ was considered statistically significant.

\section{ACKNOWLEDGMENTS}

We appreciate Drs. Kendra A. Williams and Shengyan Xiang for English language editing.

\section{CONFLICTS OF INTEREST}

The authors have declared that no competing interests exist.

\section{GRANT SUPPORT}

This study is supported by Beijing Health Development Project \#2011-1009-02, National Natural Science Foundation of China \#81402031, Beijing Sciences and Technology Programs Z121107009212024 and Z151100001615022.

\section{REFERENCES}

1. Hegi ME, Diserens AC, Gorlia T, Hamou MF, de Tribolet N, Weller M, Kros JM, Hainfellner JA, Mason W, Mariani L, Bromberg JE, Hau P, Mirimanoff RO. MGMT gene silencing and benefit from temozolomide in glioblastoma. N Engl J Med. 2005; 352:997-1003.

2. Liu HW, Liu XW, Dong GY, Zhou J, Liu Y, Gao Y, Liu XY, Gu L, Sun Z, Deng D. P16 Methylation as an Early Predictor for Cancer Development From Oral Epithelial Dysplasia: A Double-blind Multicentre Prospective Study. EBioMedicine. 2015; 2:432-437.

3. Cui CH, Gan Y, Gu LK, Wilson J, Liu ZJ, Zhang BZ, Deng DJ. P16-specific DNA methylation by engineered zinc finger methyltransferase inactivates gene transcription and promotes cancer metastasis. Genome Biology. 2015; 16:252.
4. Warren JD, Xiong W, Bunker AM, Vaughn CP, Furtado LV, Roberts WL, Fang JC, Samowitz WS, Heichman KA. Septin 9 methylated DNA is a sensitive and specific blood test for colorectal cancer. BMC Med. 2011; 9:133.

5. Liu Z, Zhang J, Gao Y, Pei L, Zhou J, Gu L, Zhang L, Zhu B, Hattori N, Ji J, Yuasa Y, Kim W, Ushijima T, et al. Large-scale characterization of DNA methylation changes in human gastric carcinomas with and without metastasis. Clin Cancer Res. 2014; 20:4598-4612.

6. Wang MM, Shen L, Deng DJ. Association between CHFR methylation and chemosensitivity of paclitaxel in advanced gastric cancer. Med Oncol. 2014; 31:907-908.

7. Wei JH, Haddad A, Wu KJ, Zhao HW, Kapur P, Zhang ZL, Zhao LY, Chen ZH, Zhou YY, Zhou JC, Wang B, Yu YH, Cai MY, et al. A CpG-methylation-based assay to predict survival in clear cell renal cell carcinoma. Nat Commun. 2015; 6:8699.

8. Wen L, Li J, Guo H, Liu X, Zheng S, Zhang D, Zhu W, Qu J, Guo L, Du D, Jin X, Zhang Y, Gao Y, et al. Genome-scale detection of hypermethylated $\mathrm{CpG}$ islands in circulating cell-free DNA of hepatocellular carcinoma patients. Cell Res. 2015; 25:1250-1264.

9. Herman JG, Graff JR, Myöhänen S, Nelkin BD, Baylin SB. Methylation-specific PCR: a novel PCR assay for methylation status of CpG islands. Proc Natl Acad Sci USA. 1996; 93:9821-9826.

10. Eads CA, Danenberg KD, Kawakami K, Saltz LB, Blake C, Shibata D, Danenberg PV, Laird PW. MethyLight: a highthroughput assay to measure DNA methylation. Nucleic Acids Res. 2000; 28:E32.

11. Tost J, Gut IG. DNA methylation analysis by pyrosequencing. Nat Protoc. 2007; 2:2265-2275.

12. Deng DJ, Deng GR, Smith MF, Zhou J, Xin HJ, Powell SM, $\mathrm{Lu}$ YY. Simultaneous detection of $\mathrm{CpG}$ methylation and single nucleotide polymorphism by denaturing high performance liquid chromatography. Nucleic Acids Res. 2002; 30:E13.

13. Luo DY, Zhang BZ, Lv LB, Xiang SY, Liu YH, Ji JF, et al. Methylation of $\mathrm{CpG}$ islands of $\mathrm{p} 16$ associated with progression of primary gastric carcinomas. Lab Invest. 2006; 86:591-598.

14. Grunau C, Clark SJ, Rosenthal A. Bisulfite genomic sequencing: systematic investigation of critical experimental parameters. Nucleic Acids Res. 2001; 29:E65-5.

15. Ehrich M, Zoll S, Sur S, van den Boom D. A new method for accurate assessment of DNA quality after bisulfite treatment. Nucleic Acids Res. 2007; 35:E29.

16. Deng D, Liu Z, Du Y. Epigenetic Alterations as Cancer Diagnostic, Prognostic, and Predictive Biomarkers. Adv Genet. 2010; 71:125-176.

17. Deng DJ, Lu ZM. Differentiation and Adaptation Epigenetic Networks: Translational Research in Gastric Carcinogenesis. Chin Sci Bull. 2013; 58:1-6 
18. Herman JG, Graff JR, Myöhänen S, Nelkin BD, Baylin SB. Methylation-specific PCR: a novel PCR assay for methylation status of CpG islands. PNAS. 1996; 93:9821-9826.

19. Sambrook J, Fritsch E, Maniatis T. Molecular Cloning: A Laboratory Manual. 2nd ed. Cold Spring Harbor, New York: Cold Spring Harbor Laboratory Press. 1989.

20. Eads C, Laird P. Combined bisulfite restriction analysis (COBRA). In: Mills K, Ramsahoye B, editors. DNA Methylation Protocols (Methods in Molecular Biology). Totowa, New Jersey: Humana Press. 2002; 53-70.

21. Widschwendter M, Siegmund KD, Müller HM, Fiegl H, Marth C, Müller-Holzner E, Janes PA, Laird PW.
Association of breast cancer DNA methylation profiles with hormone receptor status and response to tamoxifen. Cancer Res. 2004; 64:3807-3813.

22. Sun Y, Deng D, You WC, Bai H, Zhang L, Zhou J, Shen L, Ma JL, Xie YQ, Li JY. Methylation of p16 CpG islands associated with malignant transformation of gastric dysplasia in a population-based study. Clin Cancer Res. 2004; 10:5087-5093.

23. Cao J, Zhou J, Gao Y, Gu LK, Meng HX, Liu HW, Deng DJ. Methylation of p16 CpG Island Associated with Malignant Progression of Oral Epithelial Dysplasia: A Prospective Cohort Study. Clin Cancer Res. 2009; 15:5178-5183. 